\title{
Modelo bioeconômico para cálculo de custos e receitas em sistemas de produção de gado de corte visando à obtenção de valores econômicos de características produtivas e reprodutivas
}

\author{
João Jorge Júnior ${ }^{2}$, Vera Lucia Cardoso ${ }^{3}$, Lucia Galvão de Albuquerque ${ }^{4}$ \\ ${ }^{1}$ Auxílio financeiro CNPq - FAPESP. \\ 2 Doutorando em Produção Animal - FCAV - UNESP - Jaboticabal. \\ ${ }^{3}$ APTA Regional/SAA-SP, PRDTA Centro Leste, Ribeirão Preto, SP. \\ ${ }^{4}$ Departamento de Zootecnia - FCAV - UNESP - Jaboticabal. Pesquisadora do CNPq.
}

RESUMO - Foi desenvolvido um modelo bioeconômico para o cálculo do desempenho, dos custos e das receitas para obtenção de valores econômicos de características de interesse econômico em sistemas de produção de gado de corte no Brasil. As informações de desempenho e de parâmetros biológicos e econômicos foram obtidas em uma propriedade de gado Nelore que realiza ciclo completo com venda de reprodutores. O modelo é determinístico e estático e foram usadas planilhas Excel para a realização dos cálculos. Com base nas informações originais, foram simulados dois sistemas de produção, um fazendo o ciclo completo com venda de reprodutores (CcoR) e o outro, de cria $(\mathrm{Cc})$. Foram calculados os custos eas receitas para esses dois sistemas e, a partir disto, foram obtidos seus lucros anuais. Para o cálculo dos valores econômicos foram escolhidas as características peso à desmama (PD), peso adulto da vaca (PAV), taxa de prenhez (TP) e taxa de desmama (TD), que são de interesse nos dois sistemas de produção. Para avaliar o impacto das mudanças no desempenho das características sobre o lucro anual do sistema de produção, os valores iniciais das características foram aumentados em $1 \%$. Esse aumento resultou em mudanças positivas no lucro, observando-se que a TD foi a característica que apresentou maior impacto nos dois sistemas. Para o CcoR, os valores econômicos para PD, PAV, TP e TD foram, respectivamente, R\$ 0,40/kg, $\mathrm{R} \$ 0,09 / \mathrm{kg}, \mathrm{R} \$ 3,20 / 1 \%$ e $\mathrm{R} \$ 10,15 / 1 \%$. Para Cc, estes valores foram, respectivamente, de $\mathrm{R} \$ 1,31 / \mathrm{kg}, \mathrm{R} \$ 0,09 / \mathrm{kg}$, $\mathrm{R} \$ 2,41 / 1 \%$ e $\mathrm{R} \$ 3,36 / 1 \%$. O modelo foi capaz de reproduzir satisfatoriamente o sistema de produção de gado de corte estudado e pode ser adaptado para outras circunstâncias de produção.

Palavras-chave: ciclo completo, ciclo de cria, modelagem, Nelore, objetivos de seleção

\section{Bio-economic model to calculate costs and revenues to obtain economic values for performance traits in beef cattle production systems}

\begin{abstract}
A bio-economic model was developed to calculate costs and revenues to obtain economic values (EV) for performance traits of beef cattle in production systems in Brazil. Performance records, economic and biologic parameters were obtained from a Nelore breeding herd. Two production systems: complete breeding cycle $(\mathrm{CbcR})$ and cow-calf $(\mathrm{Cc})$ were simulated using a deterministic and static model. Costs, revenues and the annual profits were calculated using Excel ${ }^{\circledR}$ to obtain the economic values for weaning weight (WW), mature weight (MW), pregnancy rate (PR) and weaning rate (WR) in both systems. The impact of changes in genetic level of traits on annual profits of the production systems were evaluated by increasing the original values of the traits by $1 \%$. This increase resulted in positive changes on profits and the greatest impact was for WR in both systems. EV for WW, MW, PR, WR in CbcR and Cc systems were respectively R $\$ 0.40 / \mathrm{kg}, \mathrm{R} \$ 0.09 / \mathrm{kg}$, $\mathrm{R} \$ 3.20 / 1 \% ; \mathrm{R} \$ 10.15 / 1 \%$ and $\mathrm{R} \$ 1.31 / \mathrm{kg}, \mathrm{R} \$ 0.09 / \mathrm{kg}, \mathrm{R} \$ 2.41 / 1 \%$ and $\mathrm{R} \$ 3.36 / 1 \%$. The bio-economic model satisfactorily described the beef cattle production systems and can be adapted to other production circumstances.
\end{abstract}

Key Words: breeding goals, complete cycle, cow-calf cycle, modeling, Nelore

\section{Introdução}

A pecuária brasileira tem grandes condições para atender o aumento na demanda dos mercados nacional e internacional. Além de possuir o maior rebanho bovino comercial do mundo, constituído, em sua maioria, por animais azebuados, com grande capacidade de adaptação às condições de produção a pasto, o País tem disponibilidade de grandes extensões de pastagens, que possibilitam sistemas de produção extensivos, resultando em custos de produção mais baixos em comparação a outros países exportadores. 
Atualmente, existem no Brasil alguns programas de melhoramento genético envolvendo a avaliação genética de animais para diversas características de interesse econômico. Índices empíricos têm sido propostos aos criadores sem a realização de um estudo específico para a definição, em termos econômicos, dos objetivos de seleção. Éessencial o trabalho desses grupos de melhoramento nestes últimos anos, todavia, são poucos os estudos referentes à otimização do uso combinado de DEP (diferenças esperadas na progênie) para maximizar a resposta econômica à seleção.

Ponzoni \& Newman (1989) definiram objetivos de seleção como a combinação de características economicamente importantes em um sistema de produção, ou seja, são aquelas a serem melhoradas. A partir da definição dos objetivos de seleção, são escolhidos as características ou os critérios de seleção a serem usados na predição dos valores genéticos dos indivíduos.

Em um sistema de produção, avalia-se a importância econômica das características biológicas a serem incluídas com um objetivo de seleção pela obtenção de seus valores econômicos (VEs), que podem ser usados na construção de índices de seleção que proporcionem balanço ótimo das características, de acordo com a teoria do índice de seleção desenvolvida por Hazel (1943).

A modelagem é a principal ferramenta utilizada para a derivação dos valores econômicos de características de interesse mediante a aplicação de equações de lucro (profit functions) ou de modelos bioeconômicos (BEM). Esses modelos baseiam-se em análises conjuntas entre o aspecto econômico e produtivo, relacionando custos, receitas, dados biológicos e o manejo realizado na propriedade e têm sido utilizados por alguns pesquisadores (Amer et al., 1994; Hirooka et al., 1998; Tess \& Kolstad, 2000; Formigoni, 2002, entre outros) para obtenção de valores econômicos em sistemas de produção de bovinos de corte.

Werth et al. (1991) utilizaram um modelo dinâmico e estocástico para característica de reprodução e um modelo determinístico para um rebanho de cria para avaliar a influência das estratégias de gerenciamento e do desempenho reprodutivo sobre o lucro líquido do sistema de cria. De modo geral, os autores salientam a importância da simulação de diferentes situações de produção utilizando esses modelos, pois este processo ajuda os pesquisadores a identificar as áreas com maiores necessidades de pesquisa.

Um modelo determinístico foi desenvolvido por Wang \& Dickerson (1991) para simular os efeitos no ciclo de vida de ovinos em decorrência do melhoramento genético das características sob diferentes condições de manejo. Esse modelo envolveu conhecimentos anteriores para estimar a importância relativa das mudanças genéticas decorrentes dos diferentes tipos de manejo. Os valores destas mudanças podem ser usados com as estimativas de herdabilidade para desenvolver programas de melhoramento genético mais efetivos.

Tess \& Kolstad (2000) desenvolveram um modelo nos Estados Unidos para sistemas extensivos de produção de gado de corte com o objetivo de predizer o desempenho animal considerando a raça, seu estado fisiológico e a qualidade da forragem.

Hirooka et al. (1998) desenvolveram um modelo bioeconômico determinístico para descrever sistemas de produção de gado de corte no Japão visando ao desenvolvimento de objetivos de seleção e obtenção de valores econômicos. Com base em variáveis nutricionais, de manejo e econômicas, o modelo simula o ciclo de vida de vacas do rebanho e o crescimento até o abate de sua progênie, determinando a eficiência econômica e biológica do sistema. O modelo apresentou-se flexível e generalizável, podendo ser aplicado em outras circunstâncias de produção.

Amer et al. (1994), em estudo com gado de corte no Canadá, desenvolveram um modelo bioeconômico para um sistema de confinamento pelo qual compararam 11 genótipos de gado de corte ( sob condições específicas de manejo e de mercado) quanto ao ponto ideal de abate, às receitas e aos custos. Os autores relataram que o modelo pode ser usado para estabelecer um ponto ótimo ao abate nas diferentes raças e avaliar os objetivos de seleção para características de confinamento em programas de melhoramento genético.

A princípio, os modelos bioeconômicos existentes na literatura internacional para calcular o desempenho de animais, as receitas e os custos dos sistemas de produção para obtenção de valores econômicos podem ser adaptados para diferentes situações. Todavia, estes modelos são complexos e detalhados e nem sempre existe a disponibilidade das informações básicas necessárias.

Objetivou-se com este trabalho desenvolver um modelo bioeconômico para calcular os custos e as receitas de um sistema de produção de bovinos de corte e obter valores econômicos para características produtivas e reprodutivas a serem incluídas em objetivos de seleção.

\section{Material e Métodos}

As informações dos parâmetros biológicos e econômicos utilizados neste trabalho são provenientes de uma propriedade localizada na região noroeste do estado de São Paulo, que possui um rebanho de 10.500 matrizes, distribuídas em aproximadamente 8.000 ha de pastagens, formadas em sua maioria por braquiárias. Nessa propriedade, 
após o nascimento, os animais são mantidos com suas mães até a época da desmama, que ocorre em torno dos 7 meses de idade. No ato da desmama, os animais são pesados e avaliados visualmente quanto aos escores de conformação (C), precocidade (P) e musculatura (M). Com esses dados, são geradas DE (Diferenças Esperadas na Progênie) para C, P e M e DG160 (dias para ganhar 160 kg - nascimento à desmama). Ao sobreano (16 a 18 meses), são realizadas novas avaliações, considerando-se os escores de Cs, Ps e Ms, DG240 (dias para ganhar $240 \mathrm{~kg}$ - desmama ao sobreano) e PE (perímetro escrotal no sobreano).

Os machos são desmamados, em média, com $180 \mathrm{~kg}$ e as fêmeas aos $160 \mathrm{~kg}$. Logo após a desmama, os animais têm o seguinte destino:

machos: são vendidos $50 \%$ dos machos que tiveram desempenho inferior até a desmama (com base no índice empírico, calculado utilizando-se as DEP, em unidades de desvio-padrão), referente às características DG160 e C, P e M: $\mathrm{I}=((0,6 \times$ DEP DG160) $+(0,08 \times$ DEP C $)+(0,16 \times$ DEP P $)+$ $(0,16 \times$ DEP M) $)$.

O restante permanece a pasto, recebendo suplementação energética à base de sorgo $(0,45 \mathrm{~kg}$ de MS/animal/dia) por 140 dias após a desmama. Após este período, permanecem a pasto até completarem 22 meses de idade, quando se inicia o confinamento. Nesta fase, os machos são novamente classificados com base no índice empírico incluindo os desempenhos à desmama (descrito) e ao sobreano, que inclui as seguintes DEP: Cs, Ps, Ms, DG240 e PE. O índice de sobreano é:

$\mathrm{I}=((0,23 \times$ DEP DG160 $)+(0,04 \times$ DEP C $)+(0,08 \times$ DEP P $)+$ $(0,08 \times$ DEP M $))+((0,23 \times$ DEP DG 240$)+(0,04 \times$ DEP Cs $)$ $+(0,08 \times$ DEP Ps $)+(0,08 \times$ DEP Ms $)+(0,14 \times$ DEP PE $)))$

Os $50 \%$ inferiores são castrados e destinados ao confinamento para posterior abate. Os $50 \%$ superiores são submetidos a exames andrológicos; os reprovados neste exame (em torno de 1\%) são castrados e unidos ao grupo anterior, enquanto os aprovados são classificados como tourinhos e também são confinados. Os animais são submetidos ao confinamento com peso médio de $380 \mathrm{~kg}$ e permanecem durante 90 dias. São fornecidas ração concentrada e silagem de sorgo $(6,75 \mathrm{~kg}$ de MS/animal/dia de silagem de sorgo e 4,05 kg de MS/animal/dia de suplementação energética à base de sorgo) em quantidade calculada para proporcionar ganho médio diário de $1,2 \mathrm{~kg}$, de modo que os animais são abatidos com média de $490 \mathrm{~kg}$. Dos machos nãocastrados, $10 \%$ são destinados à reposição de touros da própria fazenda e os $90 \%$ restantes são comercializados em leilão no início do mês de setembro.

Fêmeas: são vendidas $10 \%$ das fêmeas que tiveram desempenhos inferiores até a desmama (com base no índice empírico, calculado utilizando-se as DEP DG160 e C, P e M da mesma forma que nos machos). O restante (90\%) permanece a pasto recebendo $0,45 \mathrm{~kg}$ de MS/animal/dia de suplementação energética à base de sorgo por 140 dias após a desmama. Ao atingirem 14 a 16 meses de idade, são vendidas mais $10 \%$ das fêmeas com desempenhos inferiores, com base no índice empírico que inclui as seguintes DEP: DG160, C, P, M, DG240, $\mathrm{Cs}$, Ps, Ms e PE (com base nas DEP dos irmãos destas fêmeas), da mesma forma que nos machos.

As fêmeas remanescentes permanecem a pasto com fornecimento de sal mineral. A estação de monta é realizada nos meses de novembro, dezembro e janeiro, sendo inseminadas todas as novilhas e $80 \%$ das vacas. Novilhas e vacas nãoprenhes são descartadas. As vacas mais velhas são abatidas e as vacas novas e novilhas são vendidas a outros criadores. Toda a venda de descarte ocorre no início do mês de maio.

Na Figura 1, são representados o fluxo entre as categorias e o destino dos animais no decorrer do ciclo de produção. Os parâmetros biológicos utilizados neste estudo estão descritos na Tabela 1 .

Após o nascimento, os animais são mantidos com suas mães até a desmama, que ocorre em torno dos 7 meses. Os machos são desmamados, em média, com $180 \mathrm{~kg}$ e as fêmeas com $160 \mathrm{~kg}$. Após a desmama, são vendidos 99\% dos machos e o restante $(1 \%)$ é destinado à reposição na fazenda. No caso das fêmeas, $15 \%$ são mantidas para reposição e o restante também é vendido logo após a desmama. O manejo reprodutivo das fêmeas neste ciclo é o mesmo que para o ciclo completo.

Na Figura 2, encontra-se um fluxograma representando a transição entre as categorias e os destinos dos animais no decorrer do ciclo de produção.

A fazenda tem como componentes anuais de receita bezerros (as) à desmama, novilhas ao sobreano, vacas de descarte, bois para abate, tourinhos e venda de sêmen (no CcoR) e bezerros(as) à desmama e novilhas e vacas de descarte (no Cc).

Os custos anuais referem-se à alimentação (silagem, suplementação energética, sal mineral), à pastagem (1/10 do custo com a formação, considerando-se que as pastagens tenham que ser renovados a cada dez anos) e à manutenção de pastagens. Foram considerados também os custos veterinários de acordo com a categoria animal (vacinações, controle de endo e ectoparasitas, exames preventivos etc) e os custos com certificação e rastreabilidade e aquisição e produção de sêmen.

O modelo bioeconômico é determinístico e estático. Os cálculos dos desempenhos produtivo e reprodutivo do rebanho, das receitas e dos custos foram realizados nas seguintes planilhas Excel: 


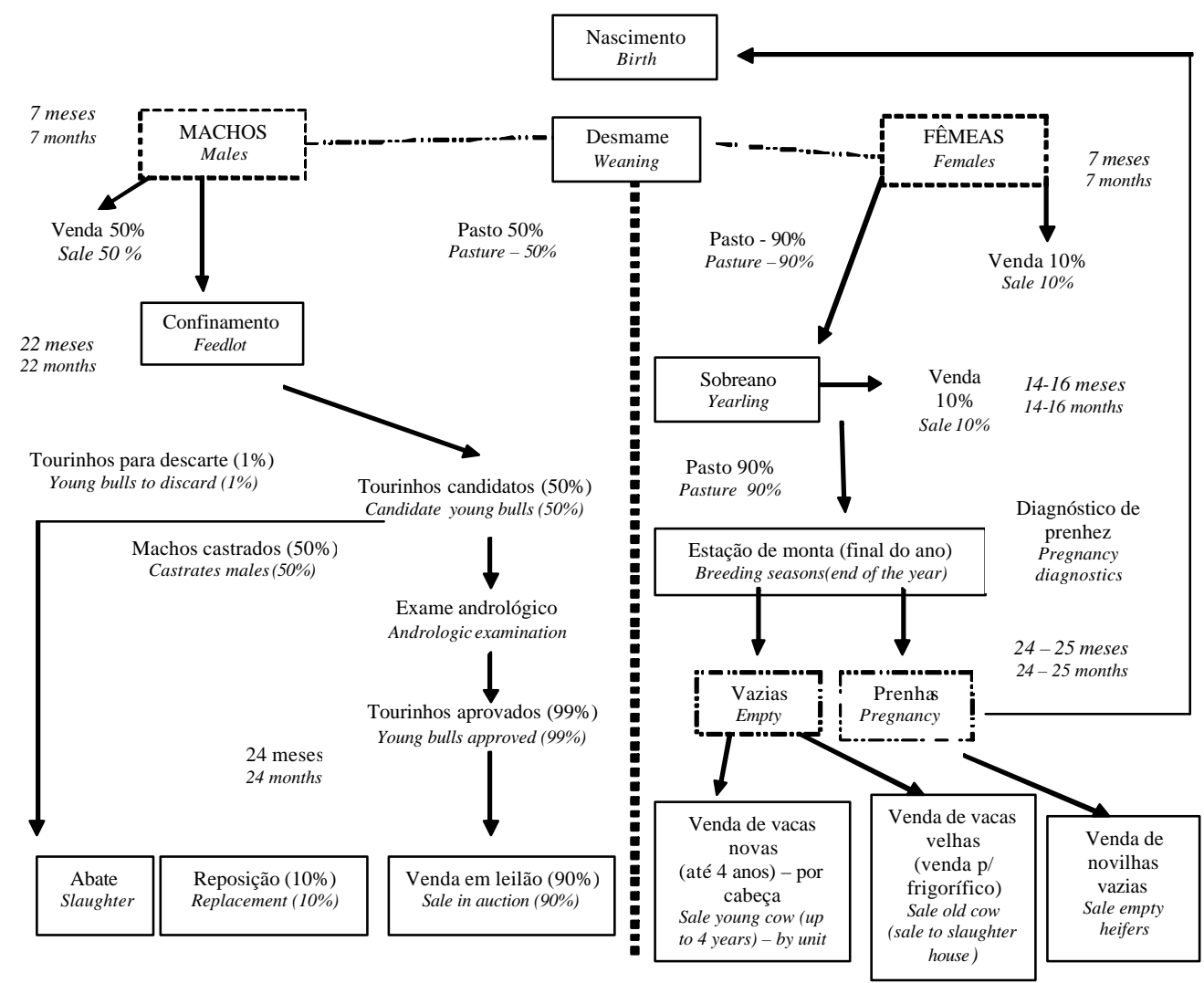

Figura 1 - Fluxo entre as categorias e destino dos animais no decorrer do ciclo completo de produção.

Figure 1 - Flow chart for categories and destination of animals during the complete breeding production cycle.

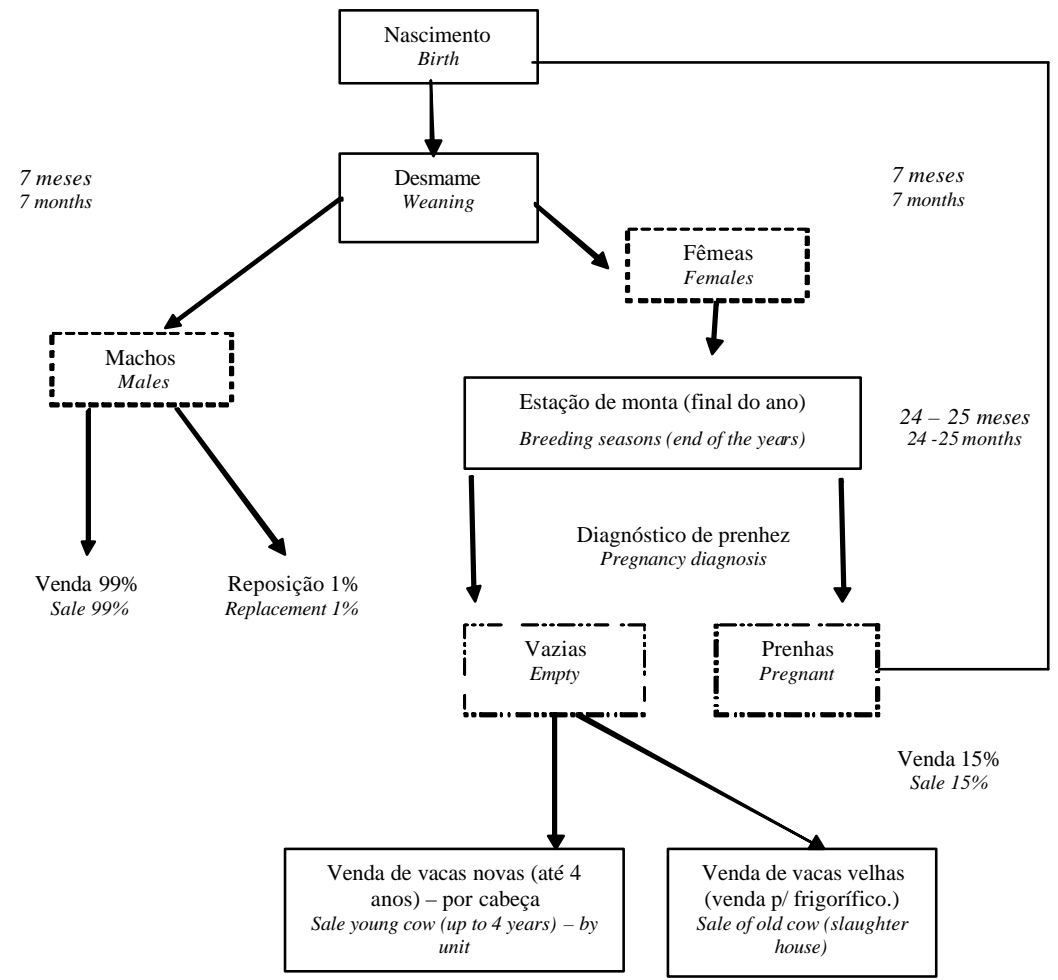

Figura 2 - Fluxo entre as categorias e destinos dos animais no decorrer do ciclo de cria de produção.

Figure 2 - Flow chart for categories and destination of animals during the cow-calf production cycle. 
Tabela 1 - Parâmetros biológicos do rebanho estudado Table 1 - Biological parameters for the herd

Parâmetro biológico

Biological parameter

Número de matrizes

Number of breeding cows

Taxa de prenhez - novilhas (\%)

Pregnancy rate - heifers (\%)

Taxa de prenhez - vacas $(\%)$

Pregnancy rate - cows (\%)

Taxa de reposição $(\%)$

Replacement rate (\%)

Idade à primeira cobertura (meses)

Age at first calving (months)

Número de doses por vaca (IA)

Number of doses per cow (AI)

Mortalidade até a desmama (\%)

Mortality up to weaning (\%)

Idade média à desmama (meses)

Average age at weaning (months)

Peso médio da vaca adulta $(\mathrm{kg})$

Average weight of mature cow $(\mathrm{kg})$

Peso médio do bezerro à desmama $(\mathrm{kg})$

Average weight of male calf at weaning ( $\mathrm{kg}$ )

Peso médio da bezerra à desmama $(\mathrm{kg})$

Average weight of female calf at weaning $(\mathrm{kg})$

Peso médio ao abate dos machos $(\mathrm{kg})$

Average weight of males at slaughter $(\mathrm{kg})$

Idade ao abate (meses)

Age at slaughter (months)

Rendimento de carcaça (machos) (\%)

Yield grade (males) (\%)

Rendimento de carcaça (vacas) (\%)

Yield grade (cows) (\%)
10.500

85

80

25

21 a 25

, 55

4

7

450

180

160

490

24

55

48

evolução anual do rebanho: a partir de um número de matrizes, a evolução anual do rebanho foi feita com base nas taxas de prenhez de vaca e novilhas; nas taxas de mortalidade de bezerros(as) até a desmama; nos números de animais vendidos à desmama e ao sobreano, de machos para abate e leilão e de fêmeas descartadas; nas taxas de prenhez de vacas e novilhas; e no número de doses/vaca utilizadas na inseminação artificial;

indices de desempenho ponderal: nesta planilha, foram incluídos os pesos e os ganhos médios diários das diferentes categorias animais, além do rendimento de carcaça dos animais destinados ao abate;

alimentação: nesta planilha, incluiu-se a ingestão (kg de MS) de suplementos energéticos para bezerros(as) desmamados(as) e de suplementos energéticos e silagem para os machos confinados;

preços dos componentes de produção: nesta planilha, foram incluídos os preços dos produtos comercializados e dos componentes de custos (alimentação, custos veterinários, rastreabilidade, sêmen etc);

evolução mensal do rebanho: baseada e relacionada à planilha “Evolução do rebanho", que calculou o número de animais em cada categoria, em cada mês do ano;

pastagens: nesta planilha, foram calculados os custos totais anuais do sistema de produção com pastagens, para todas as categorias animais, considerando a área total de pastagens, a espécie forrageira e a taxa de lotação nas épocas das águas e da seca.

custos mensais: nesta planilha, foram calculados os custos mensais para cada categoria animal para cada um dos componentes dos custos de produção, ou seja, custos veterinários, sal mineral, inseminação artificial e rastreabilidade.

receita: foram apresentados as receitas de cada categoria animal e o mês de comercialização dos produtos;

lucro: nesta planilha, foram resumidos todas as receitas e os custos anuais. É possível calcular o lucro marginal decorrente da mudança no desempenho das várias características de interesse econômico (mantendo inalterado o nível das outras) e obter o valor econômico dessas características. Os valores econômicos foram calculados pela diferença entre os lucros antes e após a seleção, que resultaram no lucro marginal (LM), a partir de:

$$
\mathrm{VE}=(1 / \mathrm{n}) \times(\mathrm{LM} / \mathrm{DD})
$$

em que DD é a diferença marginal no desempenho da característica e n, o número de animais referentes a cada categoria.

Suposições do modelo:

Para o desenvolvimento deste modelo, considerou-se que:

- o sistema de produção consiste em um sistema extensivo de produção em pastagens de braquiárias com animais da raça Nelore, realizado em ciclo completo. Os animais são manejados de acordo com as seguintes categorias: vacas, bezerros e bezerras desmamados, novilhas e bezerros de sobreano, bois de engorda, tourinhos para reposição e touros em reprodução;

- a estação de monta é realizada nos meses de dezembro, janeiro e fevereiro e resulta em estações de parição nos meses de setembro, outubro e novembro;

- a inseminação artificial é feita em todas as novilhas e em $80 \%$ das vacas;

- vacas e novilhas com diagnósticos de prenhez negativa, realizado em torno de 90 dias após o término da estação de monta, são descartadas;

$-10 \%$ das vacas são descartadas anualmente com base no desempenho de sua progênie;

- as novilhas excedentes (não utilizadas na reposição do rebanho) são vendidas; 
Tabela 2 - Preços médios dos componentes de produção Table 2 - Average prices for the production components

\begin{tabular}{|c|c|}
\hline $\begin{array}{l}\text { Componentes de receita } \\
\text { Revenue components }\end{array}$ & $\begin{array}{l}\text { Valor }(\mathrm{R} \$) \\
\text { Value }(R \$)\end{array}$ \\
\hline Bezerros para leilão & 585,00 \\
\hline $\begin{array}{l}\text { Male calves to auction } \\
@ \text { boi*(@steers) }\end{array}$ & 56,00 \\
\hline $\begin{array}{l}\text { Bois para abate* } \\
\text { Steers to slaughter* }\end{array}$ & $1.006,00$ \\
\hline $\begin{array}{l}\text { Tourinhos de leilão* } \\
\text { Young bulls to sell in auction* }\end{array}$ & $4.770,00$ \\
\hline $\begin{array}{l}\text { Bezerras (venda na fazenda) ( } \mathrm{kg} \text { de peso vivo) } \\
\text { Female calves (sale in farm) ( } \mathrm{kg} \text { body weight) }\end{array}$ & 2,70 \\
\hline $\begin{array}{l}\text { Novilhas de sobreano (venda na fazenda) } \\
\text { (kg de peso vivo)* }\end{array}$ & 2,40 \\
\hline Yearling heifers (sale in farm) ( $k g$ live weight)* & \\
\hline Novilhas vazias/descarte ( $\mathrm{kg}$ de peso vivo) & 1,90 \\
\hline $\begin{array}{l}\text { Empty/heifers for culling ( } k g \text { body weight }) \\
\text { @ vacas@cows }\end{array}$ & 45,40 \\
\hline Vacas para abate & 653,76 \\
\hline $\begin{array}{l}\text { Cows to slaughter } \\
\text { Vaca descarte (para reprodução) }\end{array}$ & $1.000,00$ \\
\hline Cow to cull (for reproduction) & \\
\hline $\begin{array}{l}\text { Componente de custo } \\
\text { Cost components }\end{array}$ & $\begin{array}{c}\text { Valores }(\mathrm{R} \$) \\
\text { Values }(R \$)\end{array}$ \\
\hline Alimentação & \\
\hline Feeding & \\
\hline Pastagem - formação e manutenção (ha/ano) & 124,50 \\
\hline $\begin{array}{l}\text { Pastures - formation and maintenance (ha/year) } \\
\text { Silagem }(\mathrm{R} \$ / \mathrm{kg} \text { de } \mathrm{MS}) * \\
\text { Silage }(R \$ / \mathrm{kg} \text { of } D M)^{*}\end{array}$ & 0,14 \\
\hline $\begin{array}{l}\text { Suplementação energética - confinamento } \\
(\mathrm{R} \$ / \mathrm{kg} \text { de MS })^{*}\end{array}$ & 0,31 \\
\hline Energy supplement -feedlot $(R \$ / \mathrm{kg} \text { of } D M)^{*}$ & \\
\hline $\begin{array}{l}\text { Silagem + suplementação energética } \\
(\mathrm{R} \$ / \mathrm{kg} \text { de } \mathrm{MS})^{*}\end{array}$ & 0,15 \\
\hline Silage + energy supplement $(R \$ / \mathrm{kg} \text { of } D M)^{*}$ & \\
\hline $\begin{array}{l}\text { Suplementação energética - pós-desmama } \\
(\mathrm{R} \$ / \mathrm{kg} \text { de MS)* }\end{array}$ & 0,35 \\
\hline $\begin{array}{l}\text { Energy supplement-post-weaning }(R \$ / \mathrm{kg} \text { of } D M)^{*} \\
\text { Sal mineral }(\mathrm{kg}) \\
\text { Mineral salt }(\mathrm{kg})\end{array}$ & 0,38 \\
\hline Custos veterinários & \\
\hline Veterinary costs & \\
\hline $\begin{array}{l}\text { Bezerras até } 7 \text { meses }(\mathrm{R} \$) \\
\text { Female calves up to } 7 \text { months }(R \$)\end{array}$ & 3,73 \\
\hline Novilhas (8-18 meses) $(\mathrm{R} \$)$ & 1,86 \\
\hline $\begin{array}{l}\text { Heifers }(8-18 \text { months })(R \$) \\
\text { Novilhas }(19-23 \text { meses) }(\mathrm{R} \$)\end{array}$ & 0,84 \\
\hline Heifers (19-23 months) $(R \$)$ & \\
\hline Vacas $(\mathrm{R} \$)($ Cows, $R \$)$ & 2,34 \\
\hline Inseminação artificial ( $\mathrm{R} \$)$ & 0,65 \\
\hline $\begin{array}{l}\text { Artificial insemination }(R \$) \\
\text { Bezerros até } 7 \text { meses }(\mathrm{R} \$) \\
\text { Calves up to } 7 \text { months }(R \$)\end{array}$ & 3,18 \\
\hline $\begin{array}{l}\text { Machos }(8-21) \text { meses }(\mathrm{R} \$)^{*} \\
\text { Males }(8-21) \text { months }(R \$)^{*}\end{array}$ & 1,86 \\
\hline Outros custos & \\
\hline Other costs & \\
\hline Andrológico (R\$/animal)* & 10,00 \\
\hline Andrologic $(R \$) *$ & \\
\hline Rastreabilidade ( $\mathrm{R} \$$ /animal) & 4,50 \\
\hline Traceability $(R \$)$ & \\
\hline Dose de sêmen ( $\mathrm{R} \$)$ & 0,65 \\
\hline Dose of semen $(R \$)$ & \\
\hline
\end{tabular}

* Componentes de produção exclusivos do sistema de CcoR.

* Components exclusive of the $\mathrm{CbcR}$ production system.
- toda a venda de descarte ocorre no início do mês de maio;

- as fontes de alimento (silagem e suplementação energética) são produzidas na própria fazenda;

- a taxa de lotação da pastagem é de 1,5 UA na época da seca (meses de junho a setembro) e de 2 UA no período das águas (outubro a maio);

- todos os machos castrados são abatidos; os machos não-castrados são tourinhos a serem utilizados na reposição de touros do rebanho (10\%) e o restante é vendido em leilão;

- os custos variáveis da fazenda são aqueles influenciados pelo nível de produção dos animais, sendo basicamente os custos com alimentação, podendo ou não incluir os custos com pastagens. Os custos anuais com formação e manutenção de pastagens são considerados fixos quando o aumento do nível de produção resultante do melhoramento genético não implica necessidade de área adicional de pastagem e variáveis quando esta necessidade existe. Outras fontes de custos fixos são os custos fixos individuais, que não são afetados por mudanças marginais no desempenho individual (vacinas, identificação, registro etc);

- o tamanho do rebanho não é modificado após mudanças marginais no mérito genético das características.

Na Tabela 2 constam os preços médios dos componentes de receitas e de custos do sistema de produção.

\section{Resultados e Discussão}

A composição do rebanho, calculada a partir dos índices zootécnicos básicos obtidos na propriedade em estudo (Tabela 1), e os valores obtidos das anotações da fazenda e calculados neste trabalho podem ser observados na Tabela 3. De modo geral, o modelo foi capaz de descrever satisfatoriamente o sistema de produção em questão no que diz respeito à composição do rebanho, às receitas e aos custos.

Os modelos bioeconômicos descritos na literatura internacional desenvolvidos com a finalidade de simular o desempenho produtivo de animais de diferentes categorias, bem como suas receitas e seus custos, em um sistema de produção de gado de corte, são complexos e detalhados e requerem um número de informações acerca dos parâmetros médios populacionais, nem sempre disponíveis. No entanto, segundo Dijkhuizen et al. (1997), no desenvolvimento de um modelo desta natureza, raramente se observa disponibilidade total de informações necessárias. Assim, a complexidade de cada modelo depende, em grande parte, da disponibilidade e do detalhamento de informações. Segundo esses autores, em muitos casos, o 
desconhecimento de valores de parâmetros básicos a serem incluídos no modelo pode ser sanado pela cooperação com especialistas nas diversas disciplinas envolvidas. Na validação de um modelo, considera-se até que ponto esse modelo pode constituir uma representação suficientemente boa da realidade para atingir os propósitos para os quais foi desenvolvido. Se considerado "válido", as decisões obtidas a partir desse modelo deverão ser similares àquelas que seriam tomadas em um modelo físico de mesma natureza. Caso não possa ser considerado "válido", quaisquer conclusões derivadas a partir desse modelo são de valor duvidoso. Dois processos de validação podem ser distintos: a validação interna consiste de um processo contínuo concomitante ao desenvolvimento do modelo, que assegura que as suposições estejam de acordo com a teoria, a experiência e o conhecimento geral; e a validação externa refere-se à comparação do desempenho do modelo com o desempenho de um modelo real. Análises de sensibilidade nas quais os valores de parâmetros relevantes são sistematicamente variados em um número de situações de interesse para determinar o impacto nos resultados podem ser usadas para este fim, sendo necessário o conhecimento dos parâmetros sensíveis a serem incluídos no modelo.

Neste estudo, optou-se por desenvolver um modelo mais simplificado, porém adequado a seus objetivos. Foi possível contornar as dificuldades de escassez de determinadas informações mediante consulta a relatórios técnicos e econômicos especializados, a informações de sistemas reais de produção, ao conhecimento e à experiência de profissionais de diferentes áreas do conhecimento e aos resultados de desempenho médio das diferentes categorias de animais, para diferentes grupamentos genéticos, obtidos na literatura nacional.

Vale ressaltar que, neste trabalho, optou-se por simular um sistema de produção com base nos parâmetros obtidos em um sistema real, que não representa necessariamente outros sistemas de produção de gado de corte no Brasil. Todavia, é possível, a princípio, adaptar este modelo para outras circunstâncias de produção mediante mudanças nos seus parâmetros básicos.

No CcoR, o componente de receita com maior expressão foi a venda de tourinhos, seguida pela venda de vacas de descarte e de bois para abate. As fontes de receitas menos importantes foram a venda de bezerras à desmama, de novilhas ao sobreano e de sêmen e os custos mais significativos foram aqueles referentes à alimentação (silagem, suplementação energética e formação e manutenção de pastagens). As diferenças observadas entre os resultados obtidos pelo modelo simulado e os observados na propriedade (CcoR), relativas às receitas provenientes da venda de vacas descartadas e bezerros, foi decorrente da diferença no número de matrizes, que, no modelo, foi padronizado em 10.500, enquanto, no modelo real, foi de 10.377.

No $\mathrm{Cc}$ a receita com venda de animais à desmama teve importância relativamente maior que no CcoR, visto que o número de animais comercializados nesta época é maior que no outro ciclo. Os valores referentes à venda de vacas de descarte foram semelhantes nas duas situações.

A diferença entre o valor obtido a partir do modelo simulado e o observado na propriedade, relativa aos custos com pastagem, deve-se ao fato de que, na propriedade, há disponibilidade de pastagens de reserva, utilizadas quando necessário, enquanto, no modelo, foi calculada uma área mínima por categoria, considerando-se o número de animais e a taxa de lotação, independentemente de permanecerem durante todo o ano na mesma pastagem.

Ressalta-se que os componentes de custo foram definidos com o objetivo específico de calcular valores econômicos. Assim, outros itens que seriam considerados em planilhas de custos para sistemas de produção de gado de corte, para fins de gerenciamento, não foram incluídos por não serem necessários para o objetivo deste trabalho. Supôs-se que o rebanho é estável e que não existe restrição ao aumento da quantidade de insumos. Desse modo, o aumento do desempenho dos animais em decorrência de seleção não acarreta redução no tamanho do rebanho e, portanto, os custos fixos permanecem inalterados, não precisando ser considerados no cálculo dos valores econômicos, pois são excluídos no processo de derivação.

Os valores dos componentes de receitas e custos são influenciados pelo potencial genético dos animais para as diferentes características. Modificando-se seus valores inicias, pode-se avaliar o impacto das características sobre o lucro anual do sistema de produção. Foram escolhidas as características, peso à desmama, peso adulto da vaca, taxa de prenhez e taxa de desmama, que são de interesse nos dois sistemas de produção. Seus valores iniciais foram aumentados em $1 \%$ e seus efeitos no lucro anual do sistema, tanto no Cc quanto no CcoR, podem ser observados na Tabela 4. $\mathrm{Na}$ mesma tabela, também foram descritos os valores econômicos, considerando-se o lucro marginal (lucro após a seleção - lucro antes da seleção), o aumento da característica e o número de animais referentes à categoria estudada.

Todas as características apresentaram mudança positiva no lucro em decorrência da seleção. A característica que apresentou impacto maior nos dois sistemas foi a taxa de desmama, que determina, em grande parte, a quantidade 
Tabela 3 - Desempenho anual obtido a partir das anotações da fazenda (ano base de 2002) e calculado pelo modelo bioeconômico Table 3 - Annual performance obtained from farm records (year base of 2002) and calculated by the bio-economic-model

Dados da propriedade $(\mathrm{CcoR}) \quad$ Valores do modelo $(\mathrm{CcoR}) \quad$ Valores do modelo $(\mathrm{Cc})$ Farm data $(\mathrm{CbcR}) \quad$ Model values $(\mathrm{CbcR})$ Model values $(\mathrm{Cc})$

Composiçao do rebanho

Herd composition

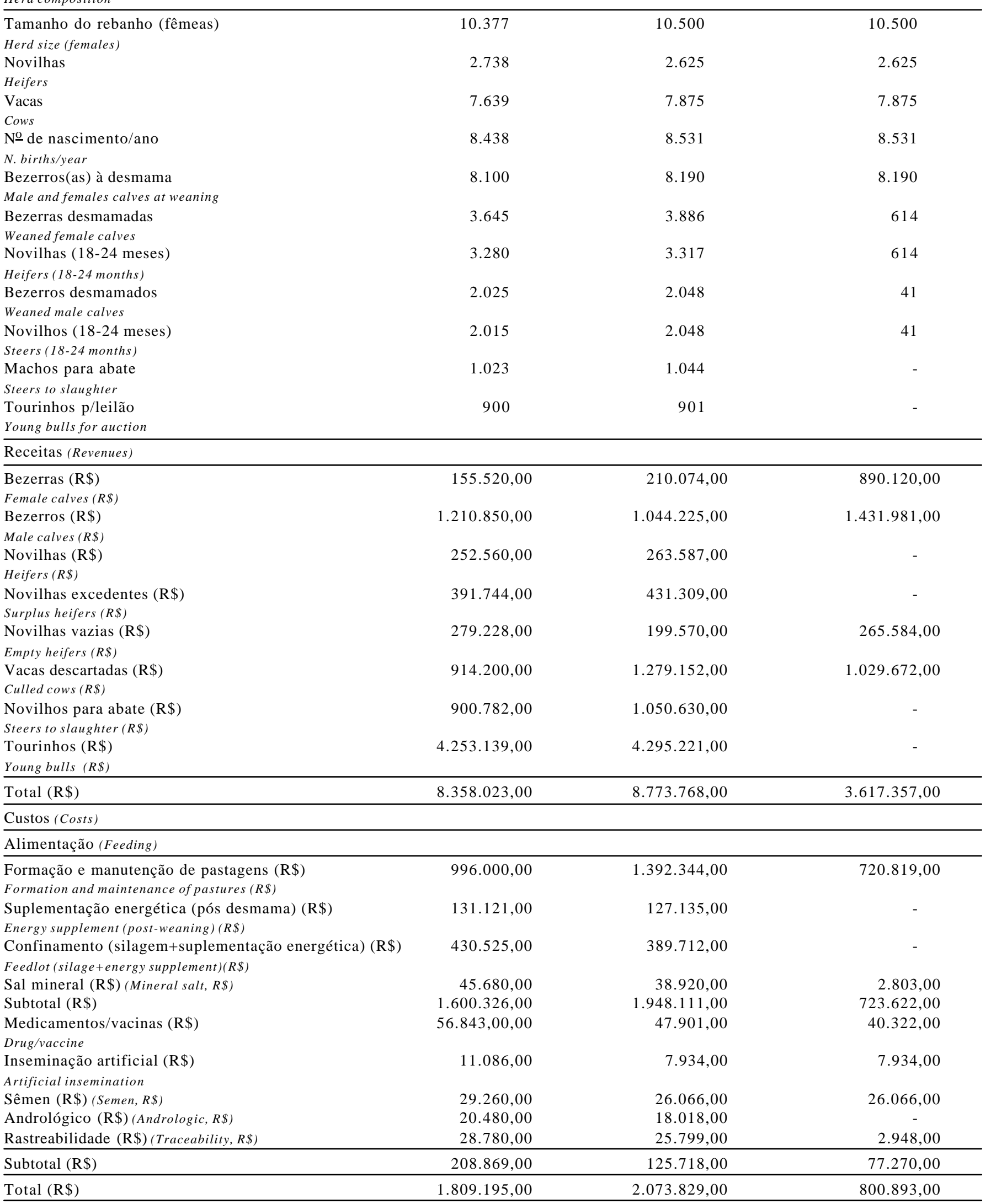


Tabela 4 - Valores das características e lucro na situação básica e depois do aumento de 1\% no desempenho das características, no ciclo completo com venda de reprodutores (CcoR) e no ciclo de cria (Cc). Valores econômicos (VE) expressos por unidade da característica

Table 4 - Trait original values and profit in the basic situation and after improvement of $1 \%$ in performance traits for the complete breeding cycle system $(\mathrm{CbcR})$ and the cow-calf system (Cc). Economic values (EV) expressed by unit of the trait

\begin{tabular}{|c|c|c|c|c|c|c|}
\hline & \multirow[t]{2}{*}{$\begin{array}{l}\text { Valor original } \\
\text { Original value }\end{array}$} & \multirow[t]{2}{*}{$\begin{array}{c}\text { Depois da mudança } \\
\text { After change }\end{array}$} & \multicolumn{2}{|c|}{$\begin{array}{l}\text { Lucro }(\mathrm{R} \$) \\
\text { Profit }(R \$)\end{array}$} & \multicolumn{2}{|c|}{$\begin{array}{l}\mathrm{VE}(\mathrm{R} \$) \\
E V(R \$)\end{array}$} \\
\hline & & & $\begin{array}{l}\mathrm{CcoR} \\
\mathrm{CbcR}\end{array}$ & $\begin{array}{l}\mathrm{Cc} \\
\mathrm{Cc}\end{array}$ & $\begin{array}{l}\mathrm{CcoR} \\
\mathrm{CbcR}\end{array}$ & $\begin{array}{l}\mathrm{Cc} \\
\mathrm{Cc}\end{array}$ \\
\hline $\begin{array}{l}\text { Situação base } \\
\text { Base situation }\end{array}$ & & & $6.899 .267,00$ & $2.816 .463,00$ & & \\
\hline Peso à desmama (kg) & 170,0 & 171,7 & $6.904 .808,00$ & $2.834 .782,00$ & $0,40 / \mathrm{kg}$ & $1,31 / \mathrm{kg}$ \\
\hline $\begin{array}{l}\text { Taxa de concepção }(\%) \\
\text { Pregnancy rate }(\%)\end{array}$ & 81,25 & 82,06 & $6.926 .503,00$ & $2.836 .993,00$ & $3,20 / 1 \%$ & $2,41 / 1 \%$ \\
\hline $\begin{array}{l}\text { Taxa de desmama }(\%) \\
\text { Weaning rate }(\%)\end{array}$ & 78,00 & 78,78 & $6.982 .413,00$ & $2.843 .979,00$ & $10,15 / 1 \%$ & $3,36 / 1 \%$ \\
\hline
\end{tabular}

de animais que serão comercializados nas várias ocasiões de venda de animais no ciclo completo e de bezerros à desmama no ciclo de cria. $\mathrm{O}$ valor econômico desta característica foi superior para o CcoR. Neste sistema, parte dos animais é comercializada ao abate e outra parte é vendida em leilões, como tourinhos de reprodução, para os quais se obtém remuneração bem superior à dos bezerros(as) vendidos ao desmame.

A taxa de concepção, que se relaciona diretamente à taxa de desmama, apresentou efeito semelhante no lucro e nas estimativas dos valores econômicos. O peso à desmama teve maior impacto no lucro no $\mathrm{Cc}$, visto que neste sistema todos os machos são comercializados nesta fase. O valor econômico desta característica foi quase quatro vezes maior que no ciclo de cria, uma vez que a maior parte da receita da fazenda é proveniente de animais vendidos à desmama e quanto maior o peso do animal, maior a remuneração. Neste caso, o valor econômico demonstra que o aumento de $1 \mathrm{~kg}$ no peso à desmama por animal acarreta aumento de $\mathrm{R} \$ 0,40$ por ano no lucro, no CcoR, e de R\$1,31, no Cc.

O peso adulto da vaca, em ambos os ciclos, afetou de forma bem discreta o lucro, resultando em valor econômico positivo, mas próximo de zero, em decorrência do baixo custo com manutenção de pastagens e do bom preço de vacas de descarte, o que significa que, a princípio, a seleção para aumento de peso em fêmeas adultas não apresentaria efeito negativo no lucro da fazenda. Todavia, salienta-se que este resultado deve ser analisado com cuidado, pois, neste estudo, supôs-se haver disponibilidade de áreas adicionais para comportar fêmeas mais pesadas em pastagens da própria fazenda, o que pode não ser realístico em longo prazo.

\section{Conclusões}

O modelo desenvolvido neste estudo foi adequado para os objetivos, pois reproduziu satisfatoriamente o sistema de produção de gado de corte estudado. Embora tenha sido desenvolvido com base em parâmetros obtidos em um sistema que não representa necessariamente outros sistemas de produção de gado de corte no Brasil, é possível adaptar este modelo a outras circunstâncias de produção mediante mudanças nos seus parâmetros básicos.

Pela alteração dos valores iniciais de cada característica, mantendo-se as outras constantes, é possível calcular as mudanças correspondentes no desempenho biológico e econômico do sistema, necessárias para o cômputo dos valores econômicos.

Entre as características para as quais se calcularam os valores econômicos, a taxa de desmame apresentou a maior importância econômica em ambos os sistemas de produção.

Para as condições assumidas neste estudo, o aumento do peso da vaca adulta não apresentaria, a princípio, impacto econômico negativo. Todavia, estudos adicionais devem ser realizados sobre esta questão, considerando outros sistemas de produção nos quais a suposição de disponibilidade de áreas adicionais de pastagem possa não ser verdadeira em longo prazo.

\section{Literatura Citada}

AMER, P.R.; KEMP, R.A.; BUCHANAN-SMITH, J.C. et al. A bio economic model for comparing beef cattle genotypes at their optimal economic slaughter end point. Journal of Animal Science, v.72, n.1, p.38-50, 1994 
DIJKHUIZEN, A.A.; JALVINGH, A.W.; HUIRNE, R.B.M. Critical steps in system simulation. In: DIJKHUIZEN, A.A.; MORRIS, R.S. (Eds.) Animal jealth economics. Principles and applications. 1.ed. Sydney: University of Sydney, 1997. p.59-67.

FORMIGONI, I.B. Estimação de valores econômicos para características componentes de índices de seleção em bovinos de corte. Pirassununga: Faculdade de Zootecnia e Engenharia de Alimentos, 2002. 79p. Dissertação (Mestrado em Zootecnia) - Universidade de São Paulo, 2002.

HAZEL, L.N. The genetic basis for constructing selection indexes. Genetics, v.28, n.2, p.476-490, 1943.

HIROOKA, H.; GROEN A.F.; HILLERS J. Developing breeding objectives for beef cattle production. 1. A bio-economic simulation model. Journal of Animal Science, v.66, n.3, p.607-621, 1998.

PONZONI, R.W.; NEWMAN, S. Developing breeding objectives for Australian beef cattle production. Animal Production, v.49, n.1, p.35-47, 1989.
TESS, M.W.; KOLSTAD, B.W. Simulation of cow-calf production systems in a range environment: I. Model development. Journal of Animal Science, v.78, n.5, p.1159-1169, 2000.

WANG, C.T.; DICKERSON G.E. A deterministic computer simulation model of live-cycle lamb and wool production. Journal of Animal Science, v.69, n.11, p.4312-4323, 1991.

WERTH, L.A.; AZZAM, S.M.; NIELSEN, M.K. et al. Use of a simulation model to evaluate the influence of reproductive performance and management decisions on net income in beef production. Journal of Animal Science, v.69, n.11, p.4170$4721,1991$. 